\title{
OTIMIZAÇÃO DE RECURSOS PARA MAXIMIZAR OS LUCROS EM UMA FÁBRICA DE CERVEJA: UTILIZAÇÃO DA PESQUISA OPERACIONAL PARA O ENCONTRO DE VALORES ÓTIMOS DE PRODUÇÃO
}

\author{
Carolina Passos Vitória Amorim \\ Universidade Veiga de Almeida \\ Rua Ibituruna, 108, Maracanã, Rio de Janeiro, RJ, CEP 20271-020 \\ Email: carol.amorim11@hotmail.com \\ Luiz Eduardo Cotta Monteiro \\ Universidade Veiga de Almeida \\ Rua Ibituruna, 108, Maracanã, Rio de Janeiro, RJ, CEP 20271-020 \\ Email: ledmontero@,hotmail.com \\ Paulo Cesar Ribas \\ Universidade Veiga de Almeida \\ Rua Ibituruna, 108, Maracanã, Rio de Janeiro, RJ, CEP 20271-020 \\ Email: paulo.ribas@petrobras.com.br
}

\begin{abstract}
Resumo
Utilizando-se do conhecimento da Pesquisa Operacional este trabalho elabora um modelo matemático para a otimização da produção e maximização dos lucros em uma fábrica de cerveja que produz três tipos diferentes de cerveja e apresenta em sua planta industrial múltiplos tanques de fermentação e maturação e uma única tina de mostura. São analisados quatro cenários com diferentes valores de capital para compra de matéria prima e as soluções são obtidas utilizando-se a interface open source Gusek.
\end{abstract}

Palavras-Chaves: Pesquisa Operacional; Fábrica de Cerveja; Otimização da produção.

\begin{abstract}
This paper, using operational research, develops a mathematical model addressed to a brewery to optimize the production plan, achieving the profit maximization. This brewery makes three different beer types, has multiple fermentation and maturation tanks and a single mashing tank. Four scenarios with different amounts of investments to purchase raw materials are analyzed. An open source solver, Gusek, is used to solve this problem.

Keywords:

Operational Research; Brewery; Production Optmization.
\end{abstract}




\section{INTRODUÇÃO}

Segundo dados divulgados no Anuário 2014 da Associação Brasileira da Indústria da Cerveja (CervBrasil) com informações da Fundação Getúlio Vargas (FGV) e do Instituto Brasileiro de Geografia e Estatística (IBGE), o setor cervejeiro responde por 2\% do PIB brasileiro e 7,8\% do PIB da indústria de transformação do país, mobiliza cerca de 12 mil fornecedores de bens e serviços e emprega 2,7 milhões de pessoas [1].

Dentro do setor cervejeiro, um novo segmento vem diversificando o mercado e ganhando destaque: o mercado de cervejas especiais. Segundo a Associação Brasileira de Bebidas [2] estima-se que existam cerca de 200 microcervejarias no Brasil e que esse segmento apresenta tendência de crescimento e deve atingir $2 \%$ da fatia do mercado de cervejas em dez anos Ainda segundo a Associação, as microcervejarias se caracterizam por serem microindústrias que têm origem familiar, com modestas instalações que permitem a produção de cerveja ou chope especial em pequenas quantidades.

Para um segmento empresarial com características de empresa familiar, é importante a necessidade das fábricas de pequeno porte se desenvolverem para se tornarem competitivas no mercado, buscando a otimização do seu processo produtivo de acordo com os diferentes cenários existentes.

O objetivo principal desse artigo consiste em fazer a análise do processo industrial de produção de cerveja em uma fábrica de pequeno porte e elaborar e desenvolver um modelo matemático que promova a otimização da produção e a maximização dos lucros da empresa. O presente modelo pode ser aplicado de forma geral ao planejamento de pequenas fábricas de cerveja devendo apenas modificar os parâmetros e algumas restrições relacionadas à demanda. Essa aplicação é possível devido ao fato de que o processo produtivo de cerveja e os equipamentos utilizados em pequenas fábricas seguem fundamentalmente etapas e características semelhantes.

\section{PESQUISA OPERACIONAL E PROGRAMAÇÃO LINEAR}

A Pesquisa Operacional surgiu durante a Segunda Guerra Mundial, na Inglaterra, ao reunir grupos multidisciplinares de físicos, matemáticos, engenheiros e cientistas sociais para encontrar soluções para problemas de grandes dimensões e complexidades, como logística, estratégia militar e utilização dos recursos militares [3].

Esses grupos multidisciplinares conquistaram grande credibilidade com o sucesso obtido no aumento da eficiência das complexas operações miliares e, após a segunda grande guerra, uma parte deles se dedicou ao meio acadêmico e ao desenvolvimento de fundamentos teóricos da pesquisa operacional e outra parte foi aplicar no meio civil as técnicas desenvolvidas nos problemas militares, trabalhando em grandes empresas e indústrias [4].

Atualmente a pesquisa operacional é uma disciplina científica de características horizontais e, seguindo seu caráter multidisciplinar, suas aplicações e contribuições estendem-se por quase todos os domínios da atividade humana, da Engenharia à Medicina, passando pela Economia e a Gestão Empresarial [5].

A Pesquisa Operacional se divide em grandes áreas, entre as quais se encontra a Programação Linear. Problemas de programação linear normalmente referem-se à distribuição eficiente de recursos limitados entre atividades competitivas com a finalidade de atender a um determinado objetivo, por exemplo, maximização de lucros ou minimização de custos. Em se tratando de programação linear, esse objetivo será expresso por uma função linear [6]. 
De acordo com Andrade, os procedimentos para a construção e desenvolvimento de modelos de otimização com o uso da Programação Linear podem ser descritos pelas seguintes etapas [7]:

A) Definição do Problema - Reconhecer que existe um problema para o qual é indicada a procura da melhor solução, pela pesquisa dos valores ótimos das variáveis de decisão.

B) Identificação das Variáveis Relevantes - Inclui: as variáveis de decisão para as quais se procura valores ótimos; variáveis exógenas que servem de base para a definição de restrições ou de variáveis endógenas; variáveis endógenas que muitas vezes entram na forma da função objetivo.

C) Formulação da função objetivo - A função objetivo expressa o critério de otimização das variáveis de decisão e deve ser expressa na forma matemática.

D) Formulação das restrições - Na maioria dos modelos de otimização, as variáveis são sujeitas a algumas restrições que devem ser expressas em formas matemáticas, e da mesma forma, o relacionamento das variáveis deve ser formulado matematicamente.

E) Escolha do método matemático de solução - Definido o problema e formulado matematicamente, deve-se escolher o método matemático apropriado para a solução do modelo.

F) Aplicação do método de solução - O método de solução é um exercício matemático que pode ser realizado manualmente ou por computador, sendo importante o conhecimento do algoritmo necessário.

G) Avaliação da solução - A solução obtida deve ser verificada e avaliada à luz das expectativas e experiências do administrador antes de ser implementada. Nessa fase do modelo pode ser necessário proceder correções e aprimoramento. Uma estimativa do risco pode ser conseguida por meio de uma análise de sensibilidade pós-otimização.

Os autores Phillips, Ravindran e Solberg apontam que a essência da pesquisa operacional reside na construção e uso de modelos e destacam algumas razões para o uso de modelos substituindo situações reais: motivações econômicas (economizar dinheiro, tempo ou algum recurso valioso); evitar riscos associados com a situação real; ajudar na comunicação e entendimento de um problema muito complexo [4] .

\section{O PROCESSO INDUSTRIAL DE FABRICAÇÃO DE CERVEJA}

O processo produtivo em uma fábrica de cerveja, de pequeno, médio ou grande porte, seguem etapas semelhantes. Alguns fatores como o tamanho do lote, a quantidade e tipos de ingredientes, a capacidade de tinas e tanques, os tipos de envase, podem apresentar variações, mas as características principais são padronizadas.

Reinold descreve o processo de produção industrial da cerveja em fábricas de pequeno e médio porte em seus artigos "Fatores Importantes para a elaboração de uma cerveja de qualidade" [8] e "A Microcervejaria e seus equipamentos" [9]. De forma resumida, o processo de produção está descrito a seguir em suas principais etapas.

Etapa 1: MOAGEM DO MALTE E MOSTURA. O processo se inicia com a moagem dos maltes de cevada para expor o amido dos grãos. Em seguida ocorre o processo de mosturação em que o malte moído é misturado com água cervejeira em temperaturas específicas de modo que ocorra a liberação de enzimas que quebram as cadeias de amido em cadeias menores de açúcares, como glicose e maltose. É feito na Tina de Mostura.

Etapa 2: CLARIFICAÇÃO. A mostura é filtrada na Tina de Clarificação, onde se separa o bagaço do mosto.

Etapa 3: FERVURA. O mosto é levado à ebulição, fervido para eliminar substâncias não desejáveis e para que seja esterilizada. Aí são adicionados ingredientes responsáveis pelo 
sabor e aroma da bebida, como por exemplo, o lúpulo. É realizada na Tina de Mostura.

Etapa 4: FERMENTAÇÃO. O mosto límpido recebe uma dosagem de levedura cervejeira. São necessários alguns dias para que os açúcares do mosto sejam consumidos pela levedura e convertidos em álcool e $\mathrm{CO}_{2}$. É feita no Tanque de Fermentação e Maturação ${ }^{1}$.

Etapa 5: MATURAÇÃO. Na maturação, algumas substâncias ainda são transformadas pela levedura em suspensão na cerveja, além de haver separação da levedura (decantação), incorporação de $\mathrm{CO} 2$ e retirada de alguns gases. É feita no Tanque de Fermentação e Maturação.

Etapa 6: FILTRAGEM. Após a maturação a cerveja passa pelo filtro de cerveja sendo armazenada no Tanque de Cerveja Pronta. Alguns estilos de cervejas não passam por esta filtração.

Etapa 7: ENVASAMENTO. A cerveja é envasada em barris, latas ou garrafas. O envasamento merece extremo cuidado para que não haja incorporação de oxigênio no interior do recipiente onde será acondicionado.

Com a crescente demanda deste produto em diversas localidades, as fábricas necessitam de fortes modernizações, e as mesmas precisam ser aplicadas tanto no maquinário quanto na qualidade do produto.

\section{ANÁLISE DO PROCESSO PRODUTIVO E DA PLANTA INDUSTRIAL}

A fábrica objeto desse estudo está situada no Estado do Rio de Janeiro e durante as visitas técnicas foram levantados dados com relação ao seu processo produtivo e realizadas estimativas e simplificações necessárias para a construção do modelo matemático para otimização da produção.

No caso analisado, a fábrica produz três tipos diferentes de cerveja:

- $\quad$ IPA (India Pale Ale) - cerveja de alta fermentação;

- GOLDEN ALE - cerveja de alta fermentação;

- $\quad$ PILSEN - cerveja de baixa fermentação.

Os ingredientes básicos na produção de cerveja consistem em água, maltes de cevada, lúpulos e leveduras. Cada tipo de cerveja tem sua receita própria e utiliza diferentes tipos e quantidades de ingredientes que irão conferir o sabor de cada uma e gerar custos diferenciados. Os custos de matéria-prima de cada tipo de cerveja são exemplificados na Tabela 1.

\begin{tabular}{|c|c|c|c|}
\hline Tipos de Cerveja & PILSEN & GOLDEN ALE & IPA \\
\hline $\begin{array}{c}\text { Custos de matéria- } \\
\text { prima }\end{array}$ & $\mathrm{R} \$ 1,00 /$ litro & $\mathrm{R} \$ 2,50 /$ litro & $\mathrm{R} \$ 5,00 /$ litro \\
\hline
\end{tabular}

Tabela 1- Tipos de cerveja e custo de matéria-prima por litro.

Analisando o processo de produção da fábrica foi identificado que não representam restrição ao processo as etapas de moagem, filtragem, envase e distribuição. Com relação aos fornecedores, os pedidos de matéria-prima são atendidos normalmente no prazo médio de uma semana, representando agilidade e conforto dentro do processo.

\footnotetext{
${ }^{1}$ Os termos "Tina de Mostura", "Tina de Clarificação" e "Tanque de Fermentação e Maturação", são os termos técnicos utilizados para descrever os respectivos equipamentos da fábrica.
} 
Foram identificados como principais gargalos dentro do processo produtivo a etapa de mostura e fervura que é realizada na Tina de Mostura, a etapa de clarificação que é feita na Tina de Clarificação e as etapas de fermentação e maturação que acontecem nos Tanques de Fermentação e Maturação. Dessa forma, as capacidades dos recursos produtivos responsáveis por essas etapas serão consideradas como importantes restrições na construção do modelo matemático. O tempo de higienização e preparação da Tina de Clarificação e da Tina de Mostura para cada produção dura o total de 10 horas e também é um fator que pode limitar o processo.

A planta industrial da fábrica analisada possui 1 Tina de Mostura com capacidade de 2000 litros, 1 Tina de Clarificação com capacidade de 2000 litros e 8 Tanques de Fermentação e Maturação com capacidade de 2000 litros cada.

O tempo de processamento nas etapas de mostura, clarificação, fervura, fermentação e maturação para produção de cada tipo de cerveja apresenta variações de tempo conforme a Tabela 2. O tempo de setup nos tanques de fermentação e maturação já estão embutidos dentro dos tempos de utilização dos respectivos tanques.

\begin{tabular}{|c|c|c|c|}
\hline Tipos de Cerveja & PILSEN & GOLDEN ALE & IPA \\
\hline MOSTURA & 1 hora & 1,5 horas & 2 horas \\
\hline CLARIFICAÇÃO & 2 horas & 2 horas & 2 horas \\
\hline FERVURA & 1 hora & 1,5 horas & 2 horas \\
\hline FERMENTAÇÃO & 6 dias & 4 dias & 3 dias \\
\hline MATURAÇÃO & 11 dias & 11 dias & 14 dias \\
\hline
\end{tabular}

Tabela 2 - Tempo de processamento para cada tipo de cerveja

O mix de produtos é fator importante para o marketing da empresa e é fundamental a produção mínima dos três diferentes tipos de cervejas de forma a atender o mercado consumidor mais próximo da fábrica, sendo que a demanda pela cerveja do tipo Pilsen é bem superior aos outros dois tipos. Com base nessa informação e no padrão de consumo para cada tipo de cerveja fez-se a estimativa da demanda mínima a ser produzida mensalmente pela fábrica. A demanda máxima de produção foi estimada considerando a previsão de consumo e o limite de capacidade de compra dos revendedores. As estimativas das demandas mínimas e máximas por mês e o lucro bruto para cada tipo de cerveja são descritos na Tabela 3.

\begin{tabular}{|c|c|c|c|}
\hline Tipos de Cerveja & PILSEN & GOLDEN ALE & IPA \\
\hline Demanda Mínima & $14,5 \mathrm{mil} 1 . / \mathrm{mês}$ & $2,0 \mathrm{mil} 1 . / \mathrm{mês}$ & $1,0 \mathrm{mil} 1 . / \mathrm{mês}$ \\
\hline Demanda Máxima & 34,5 mil $1 . /$ mês & 6,0 mil 1./ mês & 4,0 mil 1./mês \\
\hline Lucro Bruto & $\mathrm{R} \$ 2,00$ / litro & $\mathrm{R} \$ 4,00$ / litro & $\mathrm{R} \$ 7,00 /$ litro \\
\hline
\end{tabular}

Tabela 3 - Demandas e Lucro Bruto por tipo de cerveja

\section{DESCRIÇÃO DO PROBLEMA E MODELO MATEMÁTICO}

Considerando os dados levantados e as restrições identificadas no processo produtivo da fábrica, o problema consiste em encontrar os valores ótimos de produção de cada tipo de 
cerveja com o objetivo de maximizar os lucros da fábrica em um período de três meses de funcionamento.

Será considerado que a empresa possui restrição de capital somente para a compra de matéria-prima e serão feitas projeções em 4 cenários diferentes:

a) A fábrica não possui restrição de capital para compra de matéria prima.

b) A fábrica possui restrição trimestral de capital no valor de 50 mil reais para compra de matéria prima.

c) A fábrica possui restrição trimestral de capital no valor de 100 mil reais para compra de matéria prima.

d) A fábrica possui restrição trimestral de capital no valor de 150 mil reais para compra de matéria prima.

Para a construção do modelo matemático, o processo produtivo será dividido em duas fases principais: a primeira considera a sequência da mostura, clarificação e fervura que ocorrem na Tina de Mostura e na Tina de Clarificação; a segunda considera a sequência fermentação e maturação que acontece nos Tanques de Fermentação e Maturação.

A modelagem matemática do problema, com seus parâmetros e variáveis estão definidos a seguir.

A) Variáveis de decisão

$x_{1}$ - quantidade de lotes de 2000 litros de cerveja Pilsen a ser produzida

$x_{2}$ - quantidade de lotes de 2000 litros de cerveja Golden Ale a ser produzida

$x_{3}$ - quantidade de lotes de 2000 litros de cerveja IPA a ser produzida

B) Função Objetivo: maximizar o lucro.

máx $2 x_{1}+4 x_{2}+7 x_{3}$

C) Restrições:

C1) Restrição de atendimento às demandas máximas e mínimas em três meses, usando como referencia os dados da Tabela 3, segmentados em lotes de 2 mil litros. Foi utilizado o critério de arredondamento para o número inteiro imediatamente superior, considerando a exigência de números inteiros e atendimento às demandas.

$x_{1} \leq 52$

$x_{1} \geq 22$

$x_{2} \leq 9$

$x_{2} \geq 3$

$x_{3} \leq 6$

$x_{3} \geq 2$

C2) Restrição de tempo para processo de mostura, clarificação e fervura em três meses, usando como referência: a capacidade de 2000 litros da Tina de Mostura e da Tina de Clarificação; os tempos dos processos de mostura, clarificação e fervura que constam na Tabela 2; o tempo de higienização e preparo da Tina de Mostura e Tina de Clarificação que somam 10 horas para cada produção.

Considera-se o total de horas disponíveis para o processo de mostura, clarificação e fervura no período de 3 meses, funcionando 12 horas por dia e 5 dias por semana igual a 720 horas $(12 \mathrm{~h} /$ dia $\times 20$ dias $/$ mês $\times 3$ meses $=720$ horas $)$.

$14 x_{1}+15 x_{2}+16 x_{3} \leq 720$ 
C3) Restrição de tempo para o processo de fermentação e maturação em três meses, usando como referência a capacidade de 2000 litros do Tanque de Fermentação e Maturação e os tempos dos processos de fermentação e maturação que constam na Tabela 2.

Considera-se o total de horas disponíveis para o processo de fermentação e maturação em 3 meses, funcionando 24 horas por dia, 30 dias por mês, em 8 tanques, igual a: 90 dias x $8=$ 720 dias.

$17 x_{1}+15 x_{2}+17 x_{3} \leq 720$

C4) Restrição de Capital para compra de matéria-prima nos quatro cenários projetados. Serão considerados os custos de matéria-prima descritos na Tabela 1.

a) A fábrica não tem restrição de capital para compra de matéria prima.

Sem restrição.

b) A fábrica tem restrição de capital no valor de 50 mil reais para compra de matéria prima.

$2000 x_{1}+5000 x_{2}+10000 x_{3} \leq 50000$

c) A fábrica tem restrição de capital no valor de 100 mil reais para compra de matéria prima. $2000 x_{1}+5000 x_{2}+10000 x_{3} \leq 100000$

d) A fábrica tem restrição de capital no valor de 150 mil reais para compra de matéria prima. $2000 x_{1}+5000 x_{2}+10000 x_{3} \leq 150000$

\section{RESULTADOS DO MODELO MATEMÁTICO E USO DA INTERFACE GUSEK}

Para a solução do modelo matemático elaborado foi utilizado a interface Gusek. Gusek é uma interface que utiliza a linguagem GLPK - GNU Linear Programming Kit - para uso do solver open source GLP-Solve. O pacote GLPK tem o objetivo de resolver em larga escala problemas de programação linear e utiliza a linguagem MathProg modelling language [10].

O problema foi solucionado com sucesso e os valores ótimos encontrados na solução para os quatro diferentes cenários estão descritos a seguir.

a) A fábrica não tem restrição de capital para compra de matéria prima.

$x_{1}=28$ (56 mil litros de Pilsen, o que proporciona um lucro de R $\left.\$ 112.000,00\right)$

$x_{2}=9$ (18 mil litros de Golden Ale, o que proporciona um lucro de $\left.\mathrm{R} \$ 72.000,00\right)$

$x_{3}=6$ (12 mil litros de IPA, o que proporciona um lucro de $\left.\mathrm{R} \$ 84.000,00\right)$

Nessa configuração o lucro bruto trimestral é de R $\$ 268.000,00$.

b) A fábrica tem restrição de capital no valor de 50 mil reais para compra de matéria prima.

Não atende a demanda mínima.

c) A fábrica tem restrição de capital no valor de 100 mil reais para compra de matéria prima.

$x_{1}=32$ (64 mil litros de Pilsen, o que proporciona um lucro de R $\left.\$ 128.000,00\right)$

$x_{2}=3$ (6 mil litros de Golden Ale, o que proporciona um lucro de $\left.\mathrm{R} \$ 24.000,00\right)$

$x_{3}=2$ (4 mil litros de IPA, o que proporciona um lucro de $\left.\mathrm{R} \$ 28.000,00\right)$

Nessa configuração o lucro bruto trimestral é de R\$180.000,00.

d) A fábrica tem restrição de capital no valor de 150 mil reais para compra de matéria prima. 
$x_{1}=30$ (60 mil litros de Pilsen, o que proporciona um lucro de R $\left.\$ 120.000,00\right)$

$x_{2}=8$ (16 mil litros de Golden Ale, o que proporciona um lucro de $\left.\mathrm{R} \$ 64.000,00\right)$

$x_{3}=5$ (10 mil litros de IPA, o que proporciona um lucro de $\left.\mathrm{R} \$ 70.000,00\right)$

Nessa configuração o lucro bruto trimestral é de R\$254.000,00.

\section{CONCLUSÕES}

O modelo matemático foi elaborado e solucionado com sucesso podendo ser utilizado como um importante instrumento para o planejamento estratégico da empresa. Os resultados encontrados como valores ótimos são indicadores consistentes para o planejamento da produção e estabelecimento de metas da fábrica e atendem ao objetivo de maximização dos lucros e aumento da produtividade.

Os resultados encontrados nos diferentes cenários projetados revelam que diferentes valores de capital para investimento na compra de matéria-prima irão alterar significativamente os valores ótimos de produção da fábrica, alterando a proporção e o volume de produção de cada tipo de produto. Por exemplo, um capital de 100 mil reais para compra de matéria prima, para maximizar o lucro no valor de $\mathrm{R} \$ 180.000,00$, deverá produzir 64 mil litros do tipo Pilsen, 6 mil litros do tipo Golden Ale e 4 mil litros do tipo IPA, enquanto que um capital de 150 mil reais, para maximizar o lucro no valor de $\mathrm{R} \$ 254.000,00$, deverá produzir 60 mil litros do tipo Pilsen, 16 mil litros do tipo Golden Ale e 10 mil litros do tipo IPA. Nota-se que com essa variação de capital em matéria-prima, o volume do tipo Pilsen diminuiu de 64 mil litros para 60 mil litros, o volume do tipo Golden Ale aumentou de 6 mil litros para 16 mil litros e o volume do tipo IPA aumentou de 4 mil litros para 10 mil litros. Como cada tipo de cerveja utiliza diferentes tipos e quantidades de ingredientes, essa simulação tem grande utilidade para o planejamento e controle da produção de acordo com o volume de capital que se pretende investir e o modelo matemático poderá ser utilizado para simular novos cenários.

Os valores ótimos obtidos no cenário 1, em que não há restrição de capital, indicam que a restrição de demanda máxima dos tipos de cerveja Golden Ale e IPA estão atuantes. Portanto, a alteração dos parâmetros desta restrição em um cenário em que não há restrição de capital poderá gerar impacto nos valores ótimos e na lucratividade da empresa.

Em nenhum dos cenários projetados a fábrica conseguiu atingir a demanda máxima de produção o que indica que pode ser favorável o aumento da capacidade de produção da fábrica com a instalação de novos tanques de fermentação e maturação, principal gargalo do processo. Nesse sentido, o modelo matemático pode ser adaptado, simulando a instalação de novos tanques de fermentação e maturação na fábrica, possibilitando a avaliação do aumento necessário desse recurso na planta industrial de forma à atender a demanda máxima de produção.

A utilização da interface Gusek para a solução do modelo matemático se mostrou uma excelente ferramenta para resolver problemas de programação linear, e por ser parte de uma plataforma livre, a interface Gusek mostra-se uma opção acessível e eficiente para o estudante e o profissional que trabalha com esse tipo de problema.

\section{REFERÊNCIAS BIBLIOGRÁFICAS}

[1] ASSOCIAÇÃO BRASILEIRA DA INDÚSTRIA DA CERVEJA. Associação Brasileira da Indústria da Cerveja (CERVBRASIL). Site da CERVBRASIL, 03 Janeiro 2015.

Disponivel em: $<$ http://cervbrasil.org.br $>$. Acesso em: 2015 Janeiro 2015.. 
[2] ASSOCIAÇÃO BRASILEIRA DE BEBIDAS. Associação Brasileira de Bebidas (ABRABE). Site da ABRABE, 2014. Disponivel em: <www.abrabe.org.br>. Acesso em: 01 Outubro 2014.Terceira referência.

[3] PESSOA, L.; ALVES, J. M. Sistema de otimização do balanço de massas para alimentação de alto-fornos: um estudo de caso. Revista Eletrônica Produção \& Engenharia, v. 4, n. 1, Jan/Jun 2013.

[4] RAVINDRAN, A.; PHILLIPS, D. T.; SOLBERG, J. J. Operations Research: Principles and Practice. $2^{\text {a }}$. ed. New York: John Wiley \& Sons Inc, 1987.

[5] SOCIEDADE BRASILEIRA DE PESQUISA OPERACIONAL. SOBRAPO. Site da SOBRAPO, 2014. Disponivel em: <www.sobrapo.org.br>. Acesso em: 08 Dezembro 2014.

[6] PUCCINI, A. D. L. Introdução a Programação Linear. 1 ${ }^{a}$. ed. Rio de Janeiro: Ao Livro Técnico S.A, 1972.

[7] ANDRADE, E. L. D. Introdução à Pesquisa Operacional. 2a . ed. Rio de Janeiro: LTC, 2000.

[8] REINOLD, M. R. Fatores Importantes para a elaboração de uma cerveja de qualidade. Indústria de Bebidas, São Paulo, n. Ano 07 n 41, 2008.

[9] REINOLD, M. R. A Microcervejaria e seus equipamentos. Beer Life, São Paulo, n. Ano $2 \mathrm{n}^{\circ}$ 6, 2009.

[10] BETTONI, L. GUSEK. Site do GUSEK, 2015. Disponivel em: $<\mathrm{http}$ :/gusek.sourceforge.net/gusek_ptbr.html>. Acesso em: 15 jan. 2015. 